\title{
Advancement on the nondestructive evaluation of concrete using transient elastic waves
}

\author{
T.-T. Wu *, P.-L. Liu \\ Institute of Applied Mechanics, National Taiwan University, Taipei, Taiwan
}

\begin{abstract}
This paper summarizes the recent advances on the application of transient elastic waves to the nondestructive evaluation of concrete. The first part presents the numerical simulation of transient elastic waves in a plate structure with cracks and flaws. In the second part, a newly developed method for the determination of concrete elastic constants is described. The method is based on the measurements of the Rayleigh wave and the skimming longitudinal wave velocities. Instead of detecting the wave displacement normal to the specimen surface, the tangential displacement signal is detected through the utilization of home-made PZT conical transducers. Finally, recently developed methods for the detection of surface breaking cracks in concrete using transient elastic waves are summarized. The methods include the detection of surface breaking cracks using the imaging of the diffracted wave-front and the inversion technique. (O) 1998 Elsevier Science B.V.
\end{abstract}

Keywords: Transient elastic wave: NDE: Crack; Concrete: Elastic constant

\section{Introduction}

The increasing need for monitoring structure integrity has initiated intensive research in the development of nondestructive testing and evaluation methods for civil infrastructures, especially for concrete structures. Even though concrete has been utilized for many decades, it still is one of the most widely used building materials in the world. It can be found in bridges, buildings, foundations, dams, highways, etc. Unlike metallic materials that are usually assumed homogeneous, concrete consists of cement, sand and aggregates of different sizes. In addition, many microcracks or voids in concrete may exist due to the manufacturing process or external loading. The above-mentioned factors strongly affect the physical behavior of the concrete. For example, the existence of voids or microcracks in concrete decreases its compressive strength.

The use of transient elastic waves to measure the elastic properties as well as flaws of solid specimens has gained a lot of interest, especially in the nondestructive evaluation of concrete structures $[1,2]$. Since the diffraction and attenuation of elastic waves in concrete is

\footnotetext{
* Corresponding author. Fax: (886) 23639290 ;

e-mail: wutt $(w$ ndt.iam.ntu.edu.tw
}

pronounced, a relatively low frequency (large wavelength) and high energy source is usually adopted to generate elastic waves in concrete. A successful example of utilizing the transient elastic wave to the nondestructive evaluation of concrete structure in the past decade is the impact-echo method. The method was developed and applied by Carino, Sansalone and their co-workers [3-6] for the detection of flaws or voids in concrete slab and wall structures. In this method, a steel ball impact on the surface of a concrete structure is employed to generate the elastic waves. A displacement point receiver is placed next to the impact source to receive the wave signals. The fast Fourier transform (FFT) is adopted in the impact-echo method for analysis of the received wave-form. The periodic reflection of the longitudinal wave between the top surface and the flaw induces a dominated peak in the frequency spectrum and if the corresponding frequency is termed, $f$, the depth of the flaw, $D$, can be determined from the formula $D=V_{\mathrm{L}} / 2 f$.

The first step towards the development of an efficient elastic wave based nondestructive evaluation method is the study of the propagation characteristics of the elastic wave. In the literature, there are many theoretical analyses of transient elastic wave propagation in simple structures and these are of great importance in under- 
standing the elastic wave physics. To further study the diffraction waves from an arbitrary obstacle, numerical techniques such as finite difference method, finite element method and boundary integral method are of great importance. The 2-D and 3-D elastic wave propagation in heterogeneous media and concrete with crack has been investigated by $\mathrm{Wu}$ and his co-workers [7-9] using the finite difference method. The simulated results were expressed in terms of the wave signal being received at a specific point and the snapshot of the wave field at a specific time.

The elastodynamic scattering problems have played an important role in the quantitative nondestructive evaluation of materials [10]. Their solutions can be applied to develop inverse scattering techniques for flaw sizing and for modeling the reliability of flaw detection. There are analytical, numerical and experimental studies of transient elastic wave scattering by a crack in metallic structures [11-15]. The afore-mentioned studies showed that the diffracted waves from surface-breaking cracks offer sufficient information to measure the crack geometry. In addition, to extract more information about a crack, the wave-form analysis of the measured diffracted wave is suggested. Wu and Fang $[8,9]$ have successfully applied the inversion technique to determine the depth of a surface-breaking crack in concrete. Both the normal and the oblique surface-breaking cracks can be detected with this method. To measure the depth of a surfacebreaking crack in concrete, the measurement of ultrasonic wave-front has previously been employed. However, due to the large aperture of the ultrasonic transducers, the measurement of a crack in a complicaled concrete structure is not easy (if even possible). The use of the point source/point receiver method reduces the limitation and can be further utilized to obtain the imaging of the crack profile $[16,17]$.

In this paper, we summarize briefly the recent studies of numerical simulation of transient elastic wave propagation in structures with defects and the measurcment of in-situ concrete uniformity and elastic constants using transient elastic wave. The methods for the detcrmination of surface-breaking cracks in concrete structures using the transient elastic wave are also summarized.

\section{Propagation of transient elastic waves}

\subsection{Finite difference formulations}

For a body with dynamic disturbance propagating in a three dimensional space, the stress equations of motion can be written in Voigt's form as

$$
\rho \frac{\partial v_{i}}{\partial t}=T_{i j, j}+f_{i}
$$

where $\rho$ is the mass density, $f_{i}$ is the body force per unit mass, $T_{i j}$ are the stress components, $v_{i}$ are the particle velocities and $i, j=1,2,3$. For a linear elastic isotropic medium, Hooke's law reads

$\frac{\partial T_{i j}}{\partial t}=\lambda v_{k, k} \delta_{i j}+\mu\left(v_{i, j}+v_{j, i}\right)$

where $\mu$ and $\lambda$ are the Lamé constants.

To study the elastic wave propagation in a general two- or three-dimensional heterogeneous medium, the finite difference scheme with staggered grids can be adopted [18]. In this heterogeneous formulation, instead of treating the internal interfaces by explicit interfacial boundary conditions, they are represented by changing the elastic constants and mass density. The finite difference formulae for the free surface grids are derived based on the method of fictitious lines [19]. For detail of the finite difference formulation of the 2-D and 3-D elastic wave propagation problems, readers are referred to Refs. [7-9.18].

\subsection{Numerical simulation of 2-D diffracted waves}

Fig. 1(a)-(c) show snapshots $(t=6,12,18 \mu \mathrm{s})$ of the diffracted wave fields induced by a steel ball drop in an aluminum plate which contains a normal surface-break-

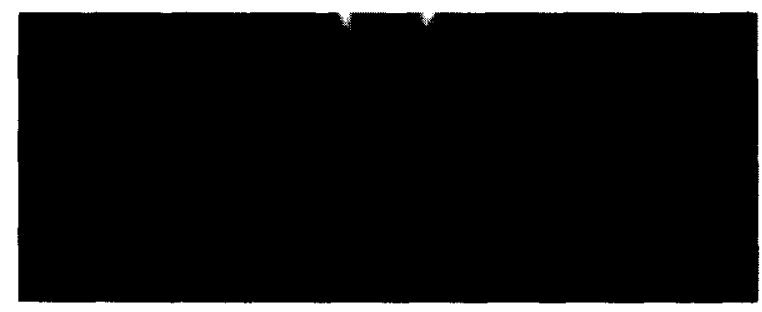

a

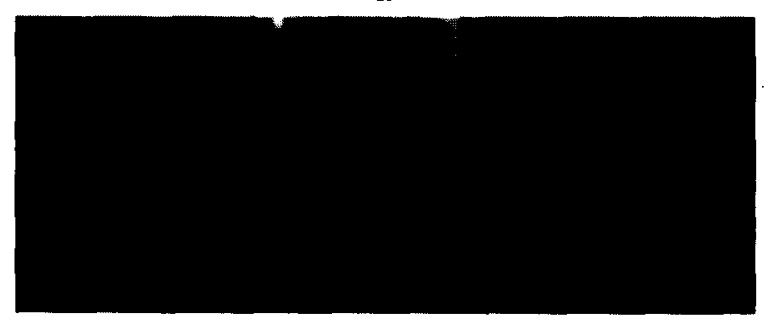

b

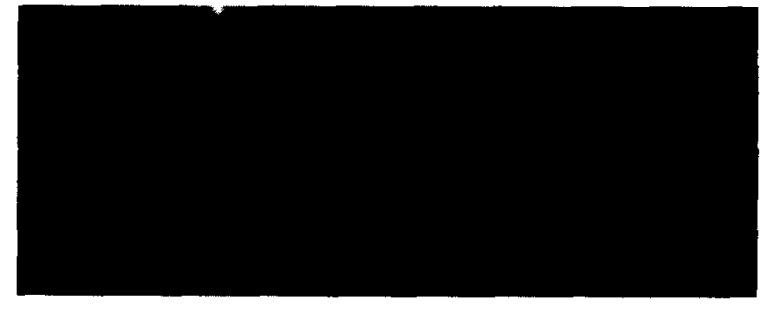

c

Fig. 1. Snapshots of the diffracted wave fields in a aluminum plate with a $2 \mathrm{~cm}$ surface-breaking crack at (a) $t=6 \mu \mathrm{s}$, (b) $t=12 \mu \mathrm{s}$, (c) $t=18 \mu \mathrm{s}$. 
ing crack. The depth of the crack and source to crack opening distances are the same and is equal to $2 \mathrm{~cm}$, the height of the plate is $8 \mathrm{~cm}$. In Fig. 1(a) $(t=6 \mu \mathrm{s})$, the Rayleigh surface waves propagating in both directions can be identified clearly, while the $\mathrm{P}$ wave-front has crept around the crack tip. In Fig. 1(b) $(t=12 \mu \mathrm{s})$, the Rayleigh surface wave which propagates toward the crack has crept down the crack. The reflected Rayleigh surface wave from the corner of the top of the crack can also be observed. In Fig. 1(c) $(t=18 \mu \mathrm{s})$, the Rayleigh wave has crept around the crack tip, and the crept $\mathbf{P}$ and $\mathbf{S}$ waves have carried wave energy to the shadow zone that located across the surface opening.

Fig. 2(a)-(c) show the snapshots $(t=6,12,18 \mu \mathrm{s})$ of the diffracted wave fields for the case of an aluminum plate with a circular hole located at the mid-depth. The diameter of the hole is $2 \mathrm{~cm}$. In Fig. 2(a) $(t=6 \mu \mathrm{s})$, one can find the $P$ wave-front is just approaching the top edge of the hole. In Fig. 2(b) $(t=12 \mu \mathrm{s})$, the $\mathrm{P}$ wavefront has passed the hole, and the $\mathrm{S}$-wave-front is arrived at the top of the hole. In addition, the scattering of the $\mathrm{P}$ wave by the hole is observed clearly. In Fig. 2(c) $(t=$ $18 \mu \mathrm{s})$, the creeping wave is formed and crept down along the boundary of the hole with a certain amount of energy. Fig. 3(a)-(c) are the snapshots $(t=6,12,18 \mu \mathrm{s})$

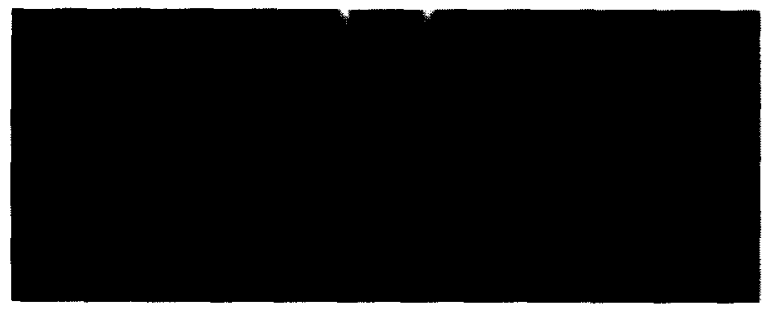

a

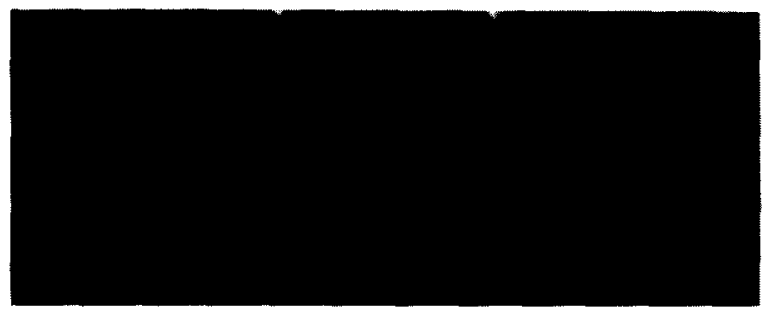

b

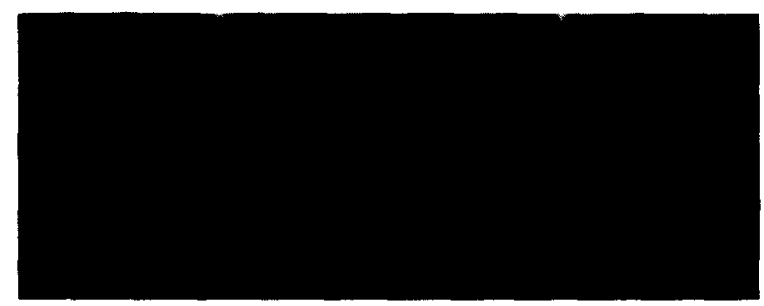

c

Fig. 2. Snapshots of the diffracted wave fields in a aluminum plate with a circular hole with $2 \mathrm{~cm}$ diameter at (a) $t=6 \mu \mathrm{s}$, (b) $t=12 \mu \mathrm{s}$, (c) $t=18 \mu \mathrm{s}$

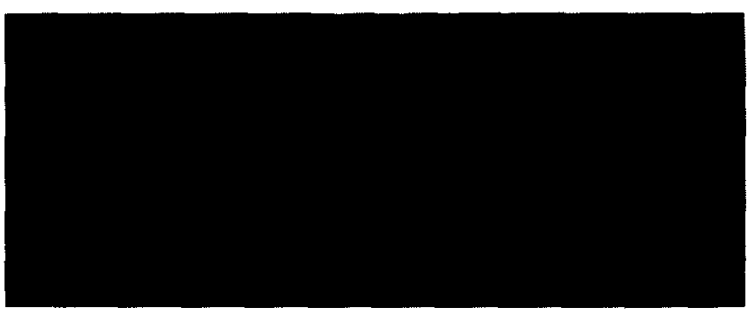

a

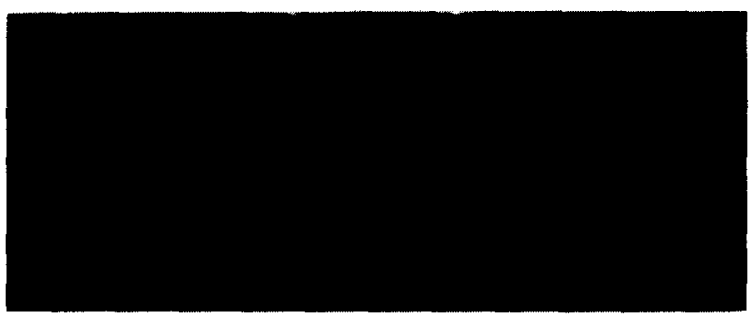

b

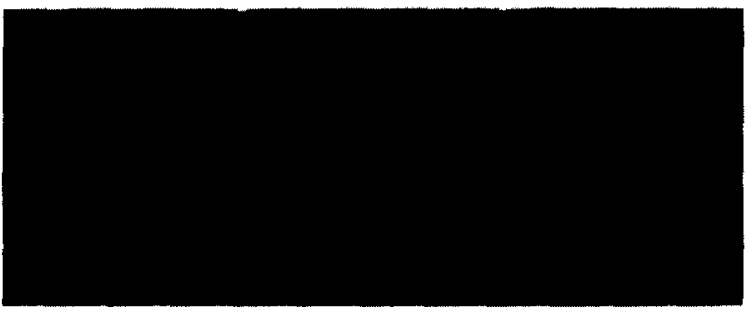

c

Fig. 3. Snapshots of the diffracted wave fields in a concrete plate with a steel bar with $2 \mathrm{~cm}$ diameter at (a) $t=6 \mu \mathrm{s}$. (b) $t=12 \mu \mathrm{s}$, (c) $t=18 \mu \mathrm{s}$.

of the diffracted fields for the case of a concrete plate with a steel bar at the mid-depth. In this case, instead of creeping around the circular boundary, the wave energy is penetrated into the steel bar and reflected back and forth in the bar.

We note that the complicated elastic wave fields scattered by an obstacle in a structure can be observed more clearly through numerically simulated snapshots. The snapshots of the scattered wave fields can be processed to obtain the dynamic image of the scaltered wave field to observe the time evolution of the various elastic wave modes.

\subsection{Numerical simulation of 3-D diffracted waves}

In this subsection, we discuss the numerical simulation of 3-D diffracted waves induced by surface-breaking cracks in concrete. Fig. 4 shows that the depth of the crack is $d$, the distance of the source and the receiver to the surface opening of the crack is $a$ and $b$, respectively. Fig. 5 shows the diffracted waves received at different receiver distances $b=2,4,6,8 \mathrm{~cm}$. The longitudinal wave and the transverse wave velocities of the concrete were assumed as $4200 \mathrm{~m} \mathrm{~s}^{-1}$ and $2450 \mathrm{~m} \mathrm{~s}^{-1}$, respectively. The source distance $a=3 \mathrm{~cm}$, the depth of the crack $d=$ $6 \mathrm{~cm}$ and the source time function is a half $\sin ^{3 / 2} t$ with a contact duration equal to $20 \mu \mathrm{s}$. From Fig. 5, we note 


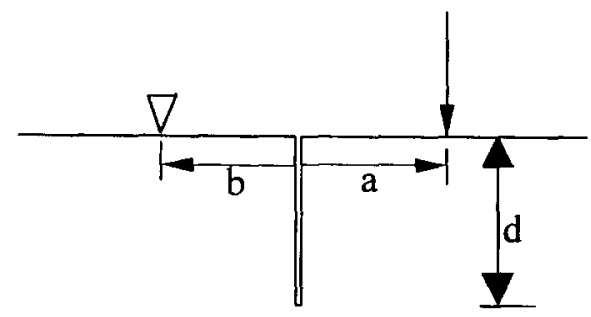

Fig. 4. The normal surface-breaking crack and arrangement of source and receiver.

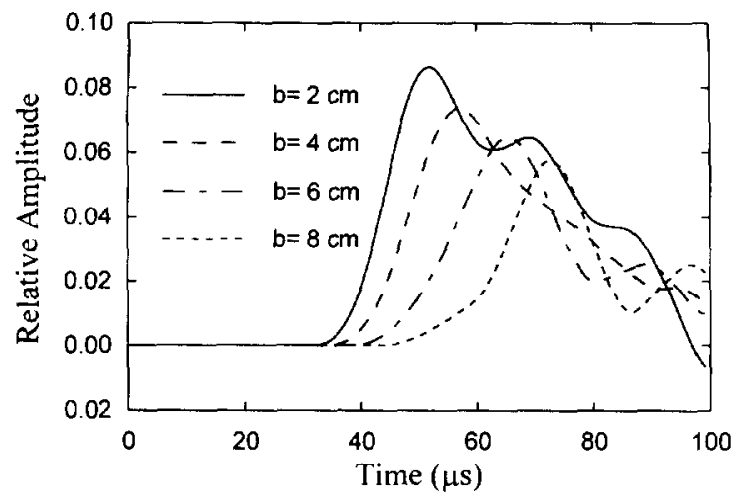

Fig. 5. The diffracted waves by a normal surface-breaking crack received at different locations $(a=3 \mathrm{~cm}$, crack depth $=6 \mathrm{~cm})[8]$.
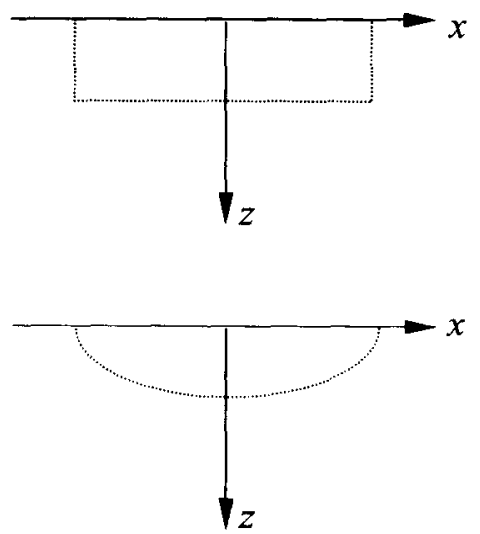

Fig. 6. The geometry of a rectangular and an elliptical surface-breaking cracks.

that the amplitude of the diffracted wave decays with the receiver distance and, in addition, the initial slope of the diffracted wave-front is also dependent on the receiver distance. It has been shown that the phase changes of the initial signal are dependent on the relative angle formed between the source to crack tip and receiver to crack tip [15].

The 3-D finite difference formulation can also be utilized to simulate the elastic wave diffraction due to cracks with different cross sections. Fig. 6 shows two different cross section of normal surface breaking cracks. The cross section of the rectangular crack is $20 \mathrm{~cm}$ in length and $5 \mathrm{~cm}$ in depth, the surface opening length of the semi-elliptical crack is $20 \mathrm{~cm}$ and the maximum depth is $5 \mathrm{~cm}$. The distance between the source and the surface opening of the crack is $2 \mathrm{~cm}$. The receiver is placed perpendicularly across the crack at a distance of $2 \mathrm{~cm}$. Similar to the ultrasonic B-scan, the B-scan images of the above two surface breaking cracks using the transient elastic waves can be obtained. The arrangement of the source and the receiver is shown in Fig. 7. The scan is along the $x$-direction from $x=-20 \mathrm{~cm}$ to $x=$ $20 \mathrm{~cm}$ with an interval of $1 / 3 \mathrm{~cm}$. The length of the time computed for each of the signal is $100 \mu$ s. On stacking all the scanning signals and utilizing the density plot, the B-scan images for the rectangular crack and elliptical crack are shown in Figs. 8 and 9, respectively. In these figures, the horizontal axis is the elapsed time and the vertical axis is the test position. In the images, the amplitude of the received signal is characterized by the

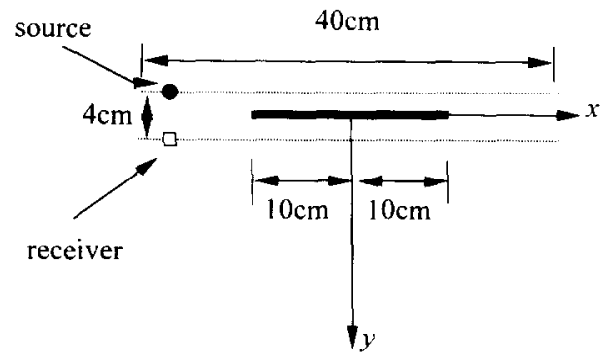

Fig. 7. The arrangement of the source and receiver for the B-scan.

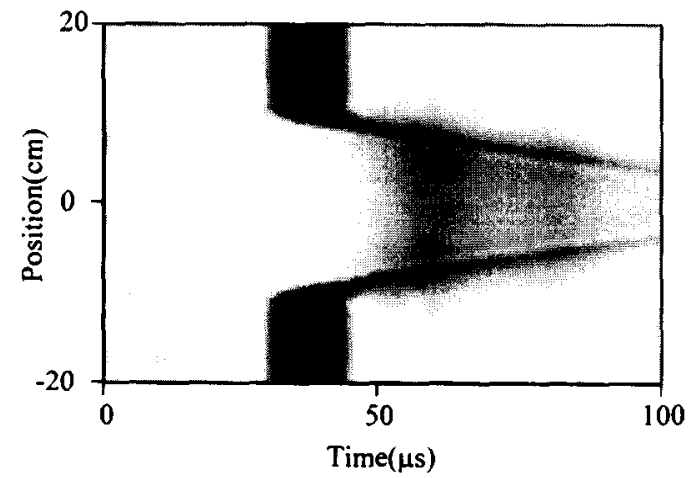

Fig. 8. B-scan image for the rectangular surface-breaking crack [8].

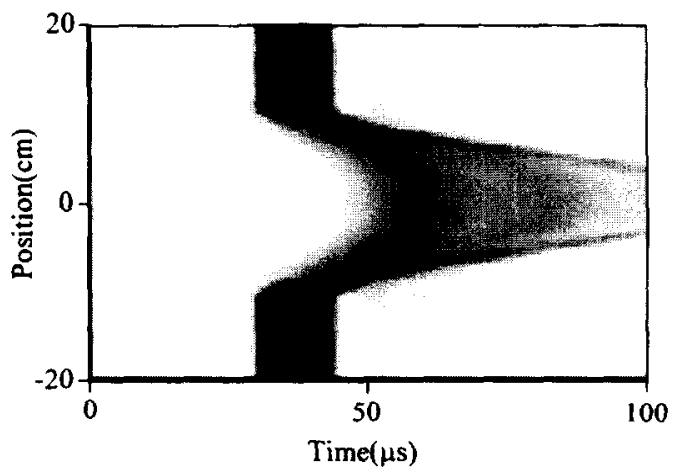

Fig. 9. B-scan image for the elliptical surface-breaking crack [8]. 
black and white contrast, i.e., the bigger the amplitude, the darker the image, the smaller the amplitude, the brighter the image. From the B-scan images, one can easily differentiate between the crack cross sections, i.e., the image of the diffracted waves from the rectangular crack is flat (Fig. 8), while that of the semi-elliptical crack is concave (Fig. 9).

\section{Measurements of concrete elastic constant}

The elastic constants of concrete are usually determined by using an uniaxial test to determine Young's modulus of a standard cylindrical specimen. In the nondestructive evaluation of concrete, the ultrasonic wave velocity is usually measured and utilized to predict the strength of concrete [20,21]. Wu et al. [22] proposed a method for determining the dynamic elastic constants of a concrete specimen using transient elastic waves. In this paper, the Rayleigh wave velocity was determined based on the cross correlation method and the longitudinal wave velocity which was determined by measuring longitudinal wave-front arrival. The major limitation of measuring the longitudinal wave velocity of a concrete specimen by the afore-mentioned method is the requirement of the presence of a perpendicular corner. Since the longitudinal wave travels across a corner, it gives a bigger displacement jump at the longitudinal wave-front arrival when a NBS conical transducer [23] (vertically polarized and normal to the specimen surface) is used. Furthermore, in the Rayleigh wave velocity measurement, the source to receiver distance is relatively large. These limitations hinder its broader application. In a following paper, Wu and Fang [24] removed the limitations of the afore-mentioned method [22] and proposed a new method based on the measurements of horizontally polarized surface responses. In the new method, the longitudinal wave-front can be identified from the surface response directly. In addition, the source to receiver distance in determining the Rayleigh wave velocity is reduced. In the following, a brief review of the recent proposed method is given.

Analyses made by Wu and Fang [24] have shown that the horizontal component of the skimming longitudinal wave-front due to a point source is much larger than its vertical response, and this suggests the utilization of the horizontal component to detect the skimming longitudinal wave-front. Furthermore, in the determination of the Rayleigh wave velocity, the source to receiver distance can be reduced significantly when the horizontal component of the surface response is used. In their study, for the purpose of detecting the radial component of a transient elastic wave signal, two horizontally polarized conical transducers were fabricated. The tip of the conical element was about $1.5 \mathrm{~mm}$ in diameter.

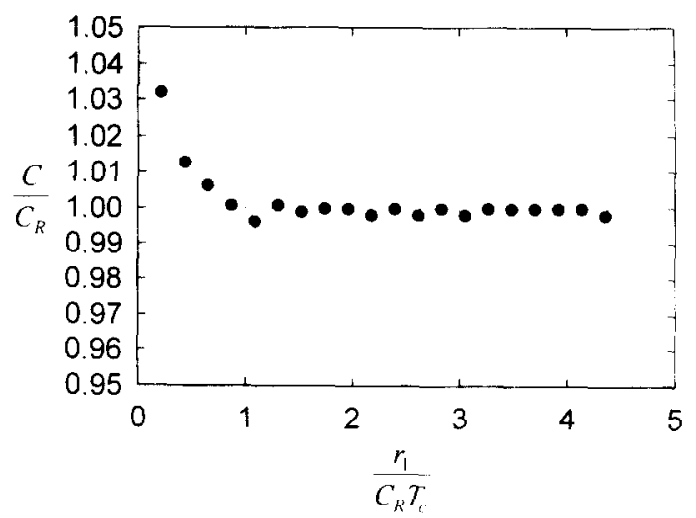

Fig. 10. The influence of $r_{1}$ on the calculated Rayleigh wave velocity using the cross correlation method (half space) [24].

In the experiment, they confirm the correctness of the afore-mentioned theoretical analyses

Wu and Fang [24] pointed out that, instead of using the vertical polarized responses, the use of horizontally polarized surface responses can reduce the source to receiver distance significantly. Fig. 10 shows the measured Rayleigh wave velocities as a function of the source to the first receiver distance $r_{1}$. In the experiment, two home-made horizontally polarized conical transducers were utilized to measure the radial component of the surface displacement signals in a concrete specimen. The received voltage signals from the conical transducers were amplified by a preamplifier and recorded by a $100 \mathrm{MHz}$ digital oscilloscope (LeCroy 9314L). The dimensions of the concrete specimen used in this study are $60 \mathrm{~cm}$ in width, $55 \mathrm{~cm}$ in length and $13 \mathrm{~cm}$ in thickness. The density of the concrete specimen is $\rho=2427 \mathrm{~kg} \mathrm{~m}^{-3}$. A steel ball $4.75 \mathrm{~mm}$ in diameter was utilized as the impact source. The measured longitudinal wave velocities of the bottom surface of the concrete plate are $4602 \mathrm{~m} \mathrm{~s}^{-1}$ and that of the top surface is $4240 \mathrm{~m} \mathrm{~s}^{-1}$. The Rayleigh wave velocities for the bottom and top surfaces of the concrete plate are $2548 \pm 28.4 \mathrm{~m} \mathrm{~s}^{-1}$ and $2302+36.2 \mathrm{~m} \mathrm{~s}^{-1}$, respectively.

With the Rayleigh wave $\left(C_{R}\right)$ and the longitudinal wave $\left(C_{1}\right)$ velocities known, the transverse wave velocity $\left(C_{7}\right)$ of the concrete specimen can be obtained by solving the well known Rayleigh wave equation, i.e.

$\left(2-\frac{C_{\mathrm{R}}^{2}}{C_{\mathrm{T}}^{2}}\right)^{2}=4\left(1-\frac{C_{\mathrm{R}}^{2}}{C_{\mathrm{L}}^{2}}\right)^{1 / 2}\left(1-\frac{C_{\mathrm{R}}^{2}}{C_{\mathrm{T}}^{2}}\right)^{1 / 2}$.

Once the longitudinal and the transverse wave velocities of an isotropic material are known, the dynamic elastic constants of the concrete specimen can be determined provided that the density of the concrete is estimated. The influence of the reinforced bars on the determination of the longitudinal wave and Rayleigh wave velocities in reinforced concrete structures is currently under study by the first author. 


\section{Concrete crack depth measurements}

Measurements of the size and geometry of a surfacebreaking crack is important in the evaluation of a existing concrete structure. The existence of surfacebreaking cracks in a concrete structure decreases its durability significantly. The position of a surface-breaking crack can easily be observed with the eye, but the determination of depth of the crack is not trivial. The inhomogeneous nature of the concrete structure prevents the utilization of high frequency ultrasonics. The use of transient elastic waves, based on the point-source/pointreceiver (PS/PR) technique [3-6], has gained a lot of interest in the field of nondestructive evaluation of concrete structures. In the detection of crack in concrete, Wu and Fang [25] utilized the phase characteristics of the diffracted wave-front to determine the depth of a normal surface-breaking crack in concrete. The measurement of the arrival of diffracted wave-front technique was utilized by Lin et al. [16] to determine the depth of a normal surface-breaking crack and by Liu et al. [17] to construct the image of surface-breaking cracks. On using the finite difference forward solution and a nonlinear optimization algorithm, the geometry of a surfacebreaking crack in concrete can be determined inversely using the measured diffracted wave signal $[8,9]$. The inverse results showed that both the depth and the inclined angle of an oblique surface-breaking crack can be accurately determined. In this paper, the wave-front imaging method and the inversion method are summarized briefly.

\subsection{Wave-front imaging}

The principle of migration in reflection seismology can be combined with the transient elastic wave measurements to construct the image of a surface-breaking crack in a concrete structure [16]. Consider a cracked halfspace, and let a signal be emitted from the source on the surface. The signal will be diffracted by the crack tip, and arrive at a receiver which is on the opposite side of the crack opening. Suppose the travel time of the diffracted signal is $t$, and the wave velocity is $v$. Then, the travel distance of the signal is $v t$. If the diffraction path is unknown, any point in the medium with the same travel distance is a possible diffraction point. Therefore, the crack tip should fall on an ellipse with the source and receiver as its foci.

A migration image can be constructed using this idea. Firstly, draw a half-plane and mark the source and receiver locations on the surface. Take a response curve and construct an ellipse for each of the data points on the curve according to the travel distance. Since the amplitude of the data points varies, the elliptic trajectories are of different magnitudes. Representing the magnitude by grayscale, a grayscale image is obtained. Since

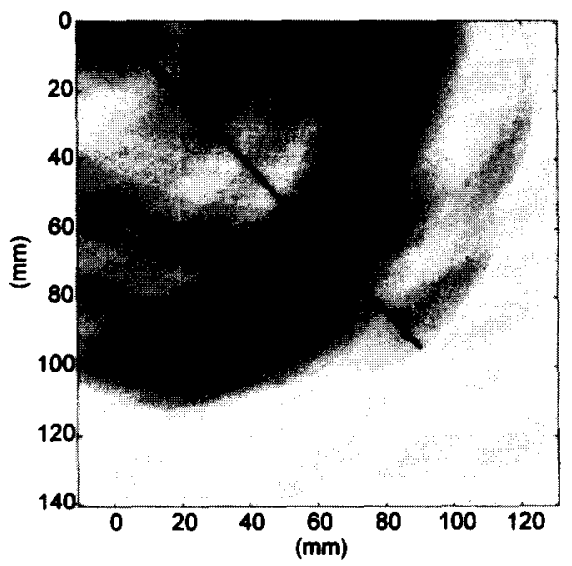

Fig. 11. Migration image of a $45^{\circ}$ model crack [16]

there is a visible change in the response curve when the first diffracted signal arrives, the ellipse of the first diffraction can be located on the image. If more than one diffraction curve migrates, the crack tip is simply located at the common intersection of the ellipses of the first arrivals.

The grayscale image obtained by migration only depicts the location of the crack tip, not the crack itself. To make the image more easily read, one can connect the crack opening and tip with a line segment in the image to represent the crack. Fig. 11 shows the experimental image of a $45^{\circ}$ crack obtained by processing the velocity signals of a concrete specimen. It is seen that the imaging method can display a real crack successfully.

\subsection{Inversion of diffracted wave}

The inversion of diffracted wave method utilizes the time domain diffracted wave signals to inversely determine the crack depth of the surface breaking crack in concrete $[8,9]$. In this method, like the impact-echo method, a steel ball is used to generate the elastic wave, and the diffracted elastic wave is received by a receiver located across the crack. A three-dimensional finite difference program is used to calculate the forward solution of the diffracted wave. Where a forward numerical solution exists, the initial part of the measured diffracted wave signal can then be utilized to recover the crack depth by using an optimization algorithm.

The arrangement of the impact source and receiver position is the same as the one shown in Fig. 4. Fig. 12 shows the comparison of the calculated (broken line) and the measured (solid line) diffracted wave signals with $a=2 \mathrm{~cm}$, and $b=2 \mathrm{~cm}$. In the figure, the measured signals have been calibrated by deconvolving the original measured signal from the transducer transfer function. The results show the accordance of the measured and the calculated diffracted wave signals.

Based on the recent study [8,9], it is noted that the initial part of the received signal contains much informa- 


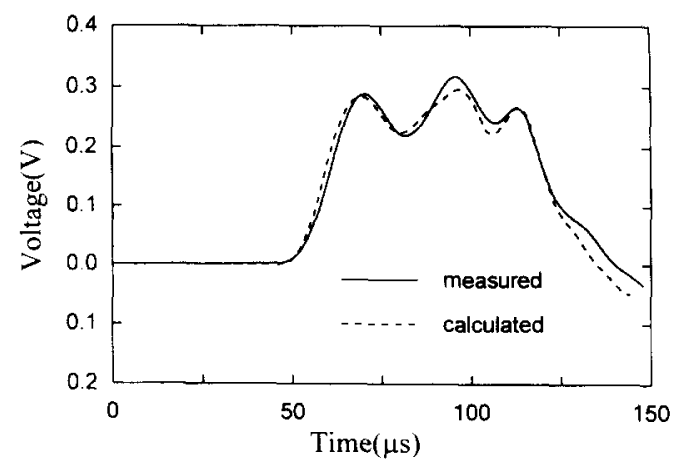

Fig. 12. Comparison of measured (solid line) and calculated (broken line) diffracted wave signals with $a=2 \mathrm{~cm}, b=2 \mathrm{~cm} \mathrm{[9].}$

tion regarding the geometry of the cracks. In addition to the arrival time and the initial phase of diffracted longitudinal wave-front, the characteristics of the later part of the diffracted wave signals were also controlled by the crack geometry. In the inversion process of the crack depth from the measured diffracted wave signal, a deviation function which defines the difference between the measured $\left(u_{\mathrm{e}}\right)$ and the guessed $\left(u_{\mathrm{g}}\right)$ normal displacement signals is defined

$e=\sum_{i=1}^{N}\left[u_{\mathrm{e}}(i)-u_{\mathrm{g}}(i) A\right]^{2}$

where $i$ represents the discrete time and $A$ is a matched constant which can be determined by the equation

$A=\frac{\sum_{i=1}^{N} u_{\mathrm{e}}(i) u_{\mathrm{g}}(i)}{\sum_{i=1}^{N} u_{\mathrm{g}}(i)-u_{\mathrm{g}}(i)}$.

In the process, three sets of initial guesses are made first, and then, the finite difference program is utilized to calculate the corresponding diffracted wave signals $\left(u_{\mathrm{g}}\right)$. With the deviation function calculated for each guessed crack tip position, the true crack tip position was then determined using the simplex method [26].

The diffracted wave signal of a normal surface-breaking crack shown in Fig. 12 was utilized as the measured signal. The depth of the crack is $9 \mathrm{~cm}$, while the source and receiver locations are $a=2 \mathrm{~cm}$ and $b=2 \mathrm{~cm}$. Fig. 13 shows a plot of the error function value versus the guessed crack depth, where $\bigcirc$ and $\times$ denote the guessed depths for two different inversion processes. In the first case $(x)$, the initial guess of the crack depth was $5 \mathrm{~cm}$. It finally converges to the true depth around $9 \mathrm{~cm}$. In the second case $(\bigcirc)$, the initial guess of the crack depth was $15 \mathrm{~cm}$. It also converges to around the truc valuc $9 \mathrm{~cm}$.

We note that the present algorithm can not only be applied to the detection of the normal surface crack, it can also be utilized to detect an oblique surface breaking crack.

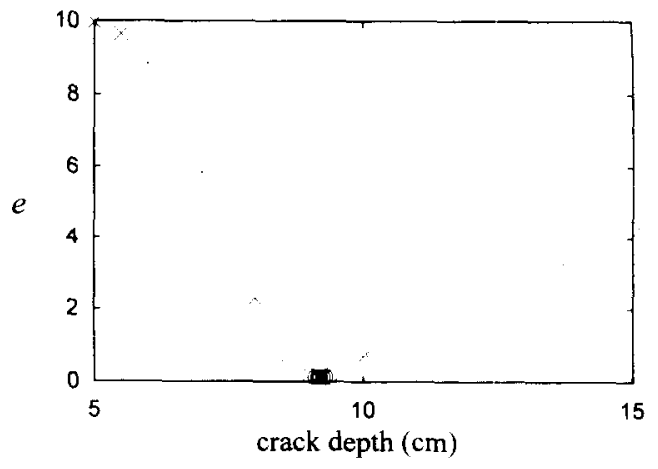

Fig. 13. The values of the error function at different crack depth [9].

\section{Concluding remarks}

To conclude this paper, we note that a successful development of an elastic wave based NDT method for testing concrete structure is strongly dependent on the solid understanding of the wave propagation characteristics. In addition to the theoretical analysis, many elastic wave-related problems require the utilization of a general purpose computer program for calculating elastic wave propagation in structures with non-simple boundary. For measuring the elastic constants or the uniformity of reinforced concrete structures, the recently proposed method that is based on the measurement of skimming longitudinal wave and Rayleigh wave velocities has demonstrated its robustness for in-situ measurement. In the detection of the depth of a surface-breaking crack in concrete, the methods described in this paper have demonstrated their feasibility through both the numerical and experimental analyses. Results of the recent applications of the crack measurement techniques in fields are very successful.

\section{Acknowledgement}

The first author thanks the financial support of this research from the National Science Council of ROC through the grant NSC-86-2621-P-002-045.

\section{References}

[1] N.J. Carino, Nondestructive testing of concrete: history and challenges, ACI Special Symposium, San Francisco, CA, 1994.

[2] V.M. Malhotra, N.J. Carino, Handbook on Nondestructive Testing of Concrete, CRC Press, Boston, MA, 1991.

[3] N.J. Carino, M. Sansalone, N.N. Hsu, A point source-point receiver pulse-echo technique for flaw detection in concrete, $\mathrm{ACI}$ J. Proc. 83 (1986) 199.

[4] M. Sansalone, N.J. Carino, Detecting honeycombing, the depth of surface-opening cracks, and ungrouted ducts using the impact echo method, Concrete International 10 (1988) 38. 
[5] Y. Lin. M. Sansalone, N.J. Carino, Finite element studies of the impact-echo response of plates containing thin layers and voids, J. Nondestructive Evaluation 9 (1) (1990) 27.

[6] N.J. Carino, M. Sansalone, Detection of voids in grouted ducts using the impact-echo method, ACI Mater. J. 89 (3) (1992) 296.

[7] T.-T. Wu, J.-H. Gong, Impact elastic waves in heterogeneous media and its application to NDE of materials, J. Acoust. Soc. Am. 94 (3) ( 1993$) 1453$.

[8] T.-T. Wu, J.-S. Fang, Inverse determination of surface-breaking cracks in concrete using transient elastic waves, Part I: Numerical forward simulation, Journal of the Chinese Institute of Civil and Hydraulic Engineering (1997) in press.

[9] J.-S. Fang, T.-T. Wu, Inverse determination of surface-breaking cracks in concrete using transient elastic waves, Part II: Experiment and inversion, J. Chinese Inst. Civil and Hydraulic Eng. (1997) in press.

[10] J.D. Achenbach, A.K. Gautesen, D.A. Mendelsohn, Ray analysis of surface-wave interaction with an edge crack, IEEE Trans. Sonics Ultrason. 27 (3) (1980) 124

[11] T. Kundu, A.K. Mal, Diffraction of elastic waves by a surface crack on a plate, J. Appl. Mech. 48 (1981) 570.

[12] C.L. Scandrett. J.D. Achenbach. Time-domain finite difference calculations for interaction of an ultrasonic wave with a surfacebreaking crack, Wave Motion 9 (1987) 171.

[13] J. Paffenholz, J.W. Fox, X. Gu, G.S. Jewett, S.K. Datta, H.A. Spetzler, Experimental and theoretical study of Rayleigh -Lamb waves in a plate containing a surface-breaking crack, Res. Nondestr. Eval. 1 (1990) 197.

[14] S.W. Liu, S.K. Datta, Scattering of ultrasonic wave by cracks in a plate, J. Appl. Mech. 60 (1993) 352.

[15] T.-T. Wu, J.-S. Fang, P.-L. Liu, Detection of the depth of a sur- face-breaking crack using transient elastic waves, J. Acoust. Soc. Am. 97 (3) (1995) 1678.

[16] Y. Lin. W.C. Su, Use of stress waves for determining the depth of surface-opening cracks in concrete structures, ACl Mater. J. 93 (5) (1996) 494.

[17] P.-L. Liu, C.-D. Tsai, T.-T. Wu, Imaging of surface-breaking concrete cracks using transient elastic waves, NDT\&E International 29 (5) (1996) 281.

[18] J. Virieux, P-SV wave propagation in heterogeneous media: velocity-stress finite-difference method. Geophysics 51 (1986) 889.

[19] Z.S. Alterman, F.C. Karal, Propagation of elastic waves in layered media by finite difference method. Bull. Seism. Soc. Am. 58 (1968) 67.

[20] Standard Test Method for Pulse Velocity Through Concrete, ASTMC597-71, 1979.

[21] A.E. Ben-Zeitun, Use of pulse velocity to predict compressive strength of concrete. Int. J. Cement Composites Light Weight Concrete 8 (1) (1986) 51.

[22] T.-T. Wu, J.-S. Fang, G.-Y. Liu, M.-K. Kuo, Determination of elastic constants of a concrete specimen using transient elastic waves, J. Acoust. Soc. Am. 98 (4) (1995) 2142.

[23] T.M. Proctor Jr... An improved piezoelectric acoustic emission transducer, J. Acoust. Soc. Am. 71 (5) (1982) 1163.

[24] T.-T. Wu, J.-S. Fang, A new method for measuring concrete elastic constants using horizontally polarized conical transducers, J. Acoust. Soc. Am. 101 (1) (1997) 330.

[25] T.-T. Wu, J.-S. Fang. Crack depth measurement of a concrete specimen using phase identification of transient elastic waves, J. Chinese Inst. Civil and Hydraulic Eng. 8 (3) (1996) 337.

[26] J.A. Nelder, R. Mead, A simplex method for function minimization. Computer Journal 7 (1965) 308. 